\title{
Performance Evaluation of an IEEE 802.15.4 Wireless Sensor Network on a Coastal Environment
}

\author{
https://doi.org/10.3991/ijim.v11i1.6315 \\ C. Flores-Cortés \\ Universidad de Colima, Colima, México \\ cfcortes@ucol.mx \\ R. Buenrostro-Mariscal \\ Universidad de Colima, Colima, México \\ raymundo@ucol.mx \\ A. Guerrero-Ibañez \\ Universidad de Colima, Colima, México \\ antonio_guerrero@ucol.mx \\ F. Estrada-Gonzalez \\ Universidad de Colima, Colima, México \\ fermin_estrada@ucol.mx \\ J. Sandoval \\ Universidad de Colima, Colima, México \\ jsandoval17@ucol.mx
}

\begin{abstract}
Wireless Sensor Networks (WSNs) have an enormous potential for investigating oceanographic problems such as the impact of industrial, touristic and commercial activities in coastal areas, among others. However, ocean waves, fog, humidity and mobility of nodes among others make difficult communication between nodes. This paper presents an on-site evaluation of the performance of an IEEE 802.15.4 WSN. In particular, the following four parameters were evaluated using different data transmission settings: received signal strength indication, throughput, round trip delay time and the rate of efficiency. Results shown that node communication between nodes on distances of up to 1.5 kilometers can be achieved but also that ocean waves did interfere with data transmission.
\end{abstract}

Keywords-wireless sensor network, IEEE 802.15.4, coastal environment, ocean observing systems, mobile technologies, performance. 


\section{Introduction}

Marine environment monitoring has become of great interest among different research groups worldwide. The efforts are focused on studying the impact of industrial, touristic and commercial activities among others on seas and oceans [1]. Because of the difficulties for collecting environmental data on these environments, there exists a growing necessity for developing Ocean Observing Systems (OOS) that facilitate deployment of infrastructure for evaluating phenomena like analysis of the quality of water in coastal areas, monitoring harmful algal bloom, conservation of species such as mangroves, whales and sea turtles, or prediction of natural disasters [2]. Depending on its objective an OOS can be composed of different components and utilize diverse architectures [3]. Examples of these components include surface buoys, gliders, autonomous underwater vehicles, coastal radars, marine sensors, ships, autonomous aerial vehicles and satellites.

Nowadays, technological advances make possible design of OOS far less complex in terms of the number and type of components utilized, more flexible, easier to configure and deploy. Examples of such systems are those based on wireless sensor networks (WSN) [3]-[7]. A key feature of WSN is communication between nodes for exchanging information. Most WSN have been designed to operate in environments other than the ocean. However, some considerations such as higher water resistance, stronger robustness, higher energy consumption, nodes mobility, among others must be taken into account for the deployment of nodes in marine environments [3]. Thus, it is important to investigate how this type of network function in these challenging environments. This paper presents preliminary results of an on-site evaluation of the IEEE 802.15.4 standard for communicating nodes within a coastal area.

To assess communication between nodes, relevant data transmission parameters such as received signal strength indication, throughput, round trip delay time and ratio of efficiency were analyzed. The four parameters were evaluated at five node transmission distances: 300, 600, 900, 1200 and 1500 meters, and five power transmission values: 7, 15, 18, 21 and $24 \mathrm{dbm}$. Because buoys move freely evaluating transmission at different distances was important. Similarly, power consumption is an important concern; this is why different power transmission settings were chosen. Our results identify the most suited settings for each distance tested. Also, when evaluating communication between nodes at distances of 1.5 kilometers, transmitting nodes had to be installed at a higher distance in relation with the sea level to successfully send and received packets. Thus, to avoid ocean interference the height at which transmitting nodes are installed must be carefully studied when designing a WSN that operates in a coastal environment.

The remainder of this paper is structured as follows. Section 2 outlines the motivation of this analysis. Section 3 details the architectural design of nodes. Several experiments were carried out using different node settings, these are discussed in section 4 . Results obtained are analyzed in section 5. Finally, conclusions and future directions are presented in section 6 . 


\section{The Case Study}

WSN based on the 802.15.4 standard have been widely studied. However, most research has focused on investigating network scalability, routing algorithms and architectures among others in environments others than the oceanographic and in most cases assessment is carried out using simulators or theoretical analysis. In [3] a review of different WSN for oceanographic monitoring is presented. Most surveyed systems do consider the challenges that oceanographic environments feature but do not evaluate on site the performance of the 802.15.4 standard. Thus, evaluation this wireless communication standard in a real scenario is an important objective of this study.

The overall motivation of this project is the design of a real-time and low cost OOS for determining and analyzing ocean current trajectories in coastal areas. Some of the main challenges of these systems are the high mobility of their nodes and the characteristics of the environment in which they are deployed. To gather the information required to identify current trajectories, nodes must navigate freely and independent of each other, this makes nodes mobility highly dynamic and difficult to predict. Also, environmental conditions must be taken into consideration because they may interfere communication between nodes.

Currently, collection of required information is carried out using drifting buoys as the one shown in Fig. 1. Each one of these buoys is fitted with a GPS device that registers its location. To collect measurements, buoys are deployed during morning and picked up on the afternoon. Then, buoys are taken to the laboratory for extracting GPS devices, exporting data to a computer and analyzing collected data. However, the whole process causes enormous delays in the analysis of the collected information, and sometimes buoys are difficult to locate because of its mobility. To simplify and make more efficient collection of data, mobile buoys will be equipped with wireless devices that will support communication between them in a wireless ad-hoc mode. When the prototype be concluded, they will communicate themselves using the protocol proposed in [7]. Wireless buoys will be equipped with sensors for collecting relevant data such as position, water temperature, salinity, $\mathrm{pH}$, among other variables. Distance between buoys at deployment will be at most 1,500 meters. Hence it is important to investigate what wireless communication technology is capable of supporting communication between nodes in a coastal environment where factors such as sea waves interfere. Results of this study will provide useful information for the physical design of buoys, as well as the power consumption requirements for supporting nodes operation. 


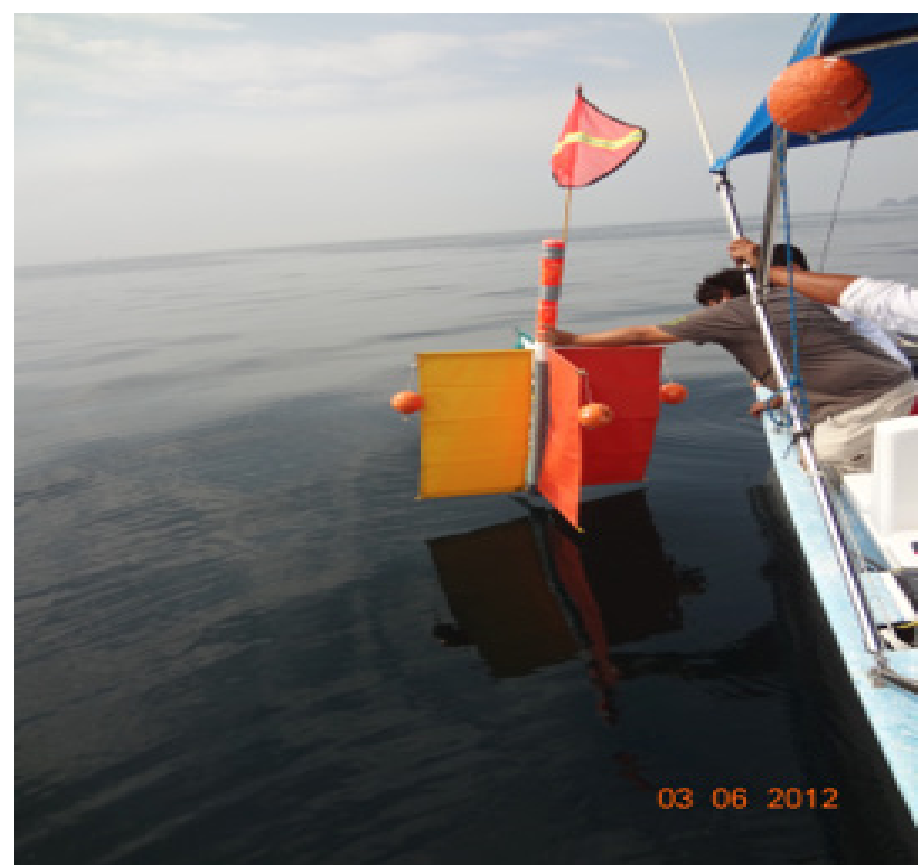

Fig. 1. A drifting buoy

\section{The Prototype Design}

To support mobile communication between nodes, XBee radios based on the IEEE 802.15.4 standard were selected. Characteristics like low cost, power consumption, transmission distance and frequency make these radios a good choice for the scenario we are targeting. This first phase of the study focused on the evaluation of radio transmission range and performance of transmissions within a coastal environment with no particular weather conditions (i.e. storm or rain). In the rest of this section considerations taken into account for the development of the prototype are described.

\subsection{Scenario and requirements}

The area we are targeting for studying ocean current trajectories is shown on Fig. 2. This coastal zone is located on Manzanillo and Santiago bays in Colima, México. Coverage area is a rectangle of $10 \mathrm{x} 4$ kilometers where environmental conditions are characterized for high levels of humidity. Thus, to design an OOS than can be deployed in scenarios like the one described, it is important to investigate what the maximum area of coverage is, the maximum distance between nodes, the weather conditions that affect wireless communications, the movement and speed of nodes and the number of nodes required. 
The following requirements were set by the oceanographic scientist team responsible of deploying the buoys and analyzing collected data:

1. Buoys are deployed in groups with a distance between nodes of 60-100 meters;

2. Buoys move freely and therefore distances between them vary;

3. Distance between groups must be at most 1.5 kilometers;

4. Data collected by each node must be at least water temperature and geospatial position;

5. Energy autonomy for up to 10 hours; and

6 . Frequency of data collection must be configurable.

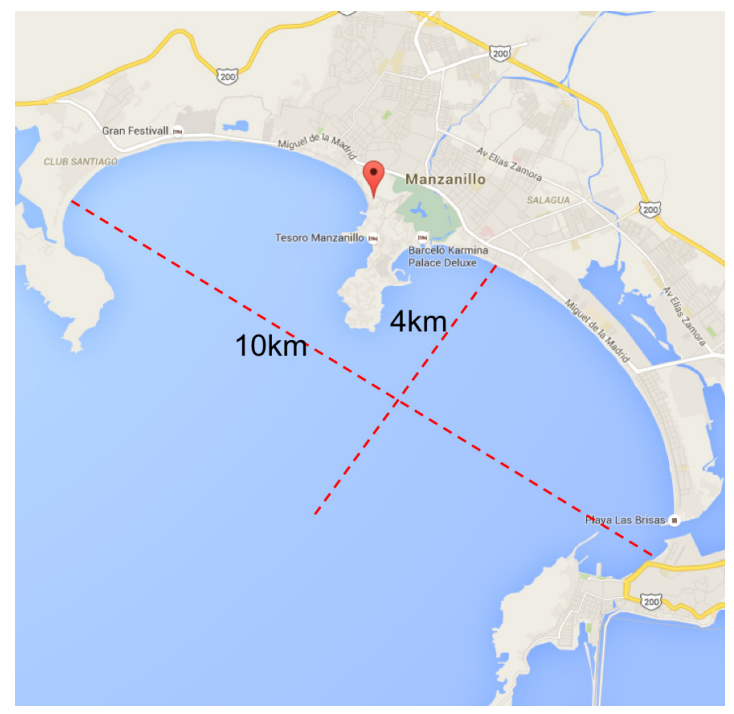

Fig. 2. Area selected for the study

\subsection{Prototype Architecture}

For evaluating wireless communication, three node configurations were assessed. The first configuration utilizes a commercial wireless sensor mote e.g. Kinetis KW40Z and Libelium WaspMote, however when compared with other options these modules are generally more expensive and have less processing power.

Another configuration features a single board computer (SBC) like BeagleBoard or Raspberry $\mathrm{Pi}$, nonetheless the main drawback of this type of devices is that they feature high levels of energy consumption and higher cost. Lastly, the use of a microcontroller device was also evaluated, these microcontrollers feature low levels of energy consumption, are highly configurable and are not expensive. Therefore, these results indicate that the best setting are microcontroller-based solutions (Arduino and NodeMCU are some examples). As a consequence we have chosen this option for our study. Table 1 lists the main characteristics of the three configurations evaluated. 
Table 1. Node configurations evaluated

\begin{tabular}{|l|l|l|l|}
\hline \multicolumn{1}{|c|}{ Feature } & \multicolumn{1}{|c|}{ Motes } & \multicolumn{1}{|c|}{ SBCs } & \multicolumn{1}{c|}{ Microcontrollers } \\
\hline Wireless radio & Included & Not included & Not included \\
Battery powered & Yes & No & Yes \\
Operating system & Yes & Yes & No \\
Energy Consumption & Low & High & Low \\
Cost & High & Low & Low \\
Processing power & Low & High & Low \\
\hline
\end{tabular}

Another configuration features a single board computer (SBC) like BeagleBoard or Raspberry $\mathrm{Pi}$, nonetheless the main drawback of this type of devices is that they feature high levels of energy consumption and higher cost. Lastly, the use of a microcontroller device was also evaluated, these microcontrollers feature low levels of energy consumption, are highly configurable and are not expensive. Therefore, these results indicate that the best setting are microcontroller-based solutions (Arduino and NodeMCU are some examples). As a consequence we have chosen this option for our study. Table 1 lists the main characteristics of the three configurations evaluated.

Fig. 3 shows a block diagram of the node configuration. The microcontroller module consists of an Arduino UNO board to manage its functionality; the location module is used to determine its location and consists of a GPS shield-v16, that has a $2.5 \mathrm{~m}$ positional accuracy; for the acquisition module a digital temperature sensor i.e. DS18B20 to measure water temperature was chosen; and finally, for the communication radio an XBee Pro 900 radio for sending information gathered to other nodes was used.

Fig. 4 shows in detail the schematic diagram which contains the power connections and data node prototype, according to the previously selected components.

Finally, Fig. 5 shows the prototype of the embedded node, according to the previously selected component, and his plastic encapsulation which was used for the performance experiments.

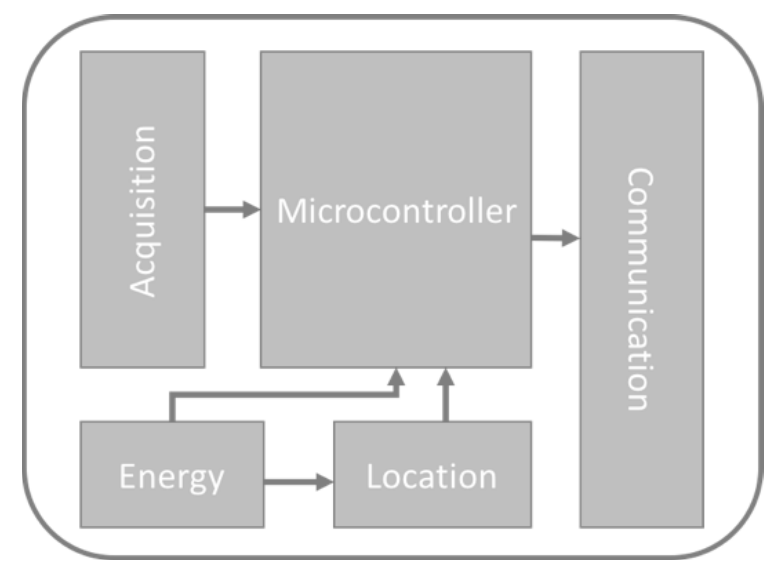

Fig. 3. Node schematic diagram 
Paper-Performance Evaluation of an IEEE 802.15.4 Wireless Sensor Network on a Coastal Environment

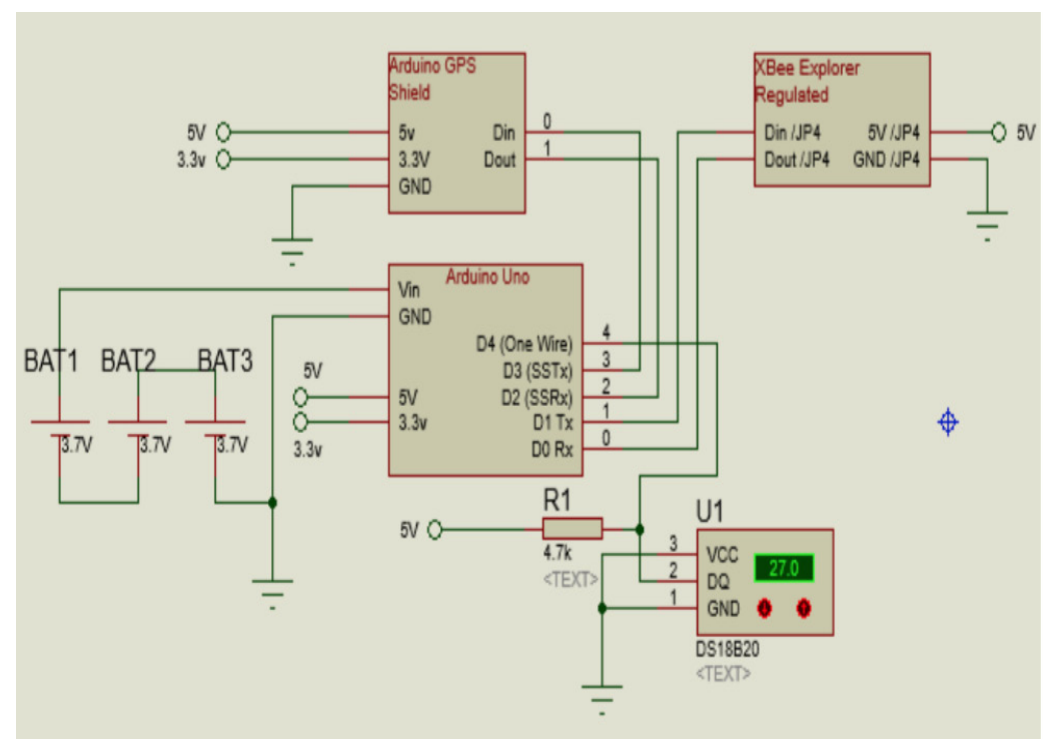

Fig. 4. Schematic diagram of the prototype node.

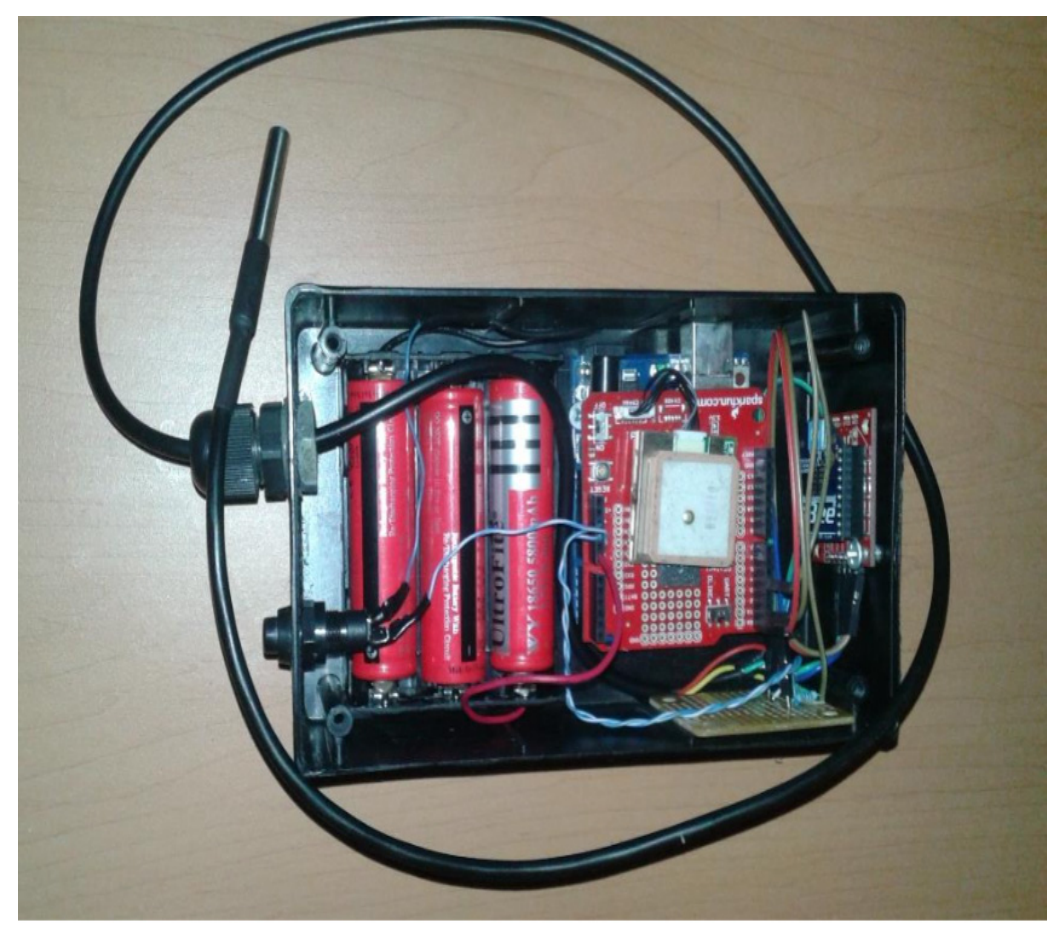

Fig. 5. Physical prototype node. 


\section{$4 \quad$ Evaluation}

In this section the design of experiments to measure the performance of the radio transmitter of the node is presented and the results obtained are analyzed.

\subsection{Experiments setup}

One of the objectives of this study is to investigate the performance of the radio transmitter in coastal areas; where there are particular climatic factors such as wind, fog and water evaporation, which can greatly degrade the quality of transmitted signals (due to problems of attenuation, refraction, reflection and scattering) [8]-[10]. Consequently, this environment can significantly reduce the theoretical range of the radio transmitter and the rate of successfully packets sent between nodes. Specifically, the study focuses on evaluating the transmission performance between nodes in a range of coverage of at least 1.5 kilometers within a typical coastal zone.

As result of the foregoing, the experiment was set up to evaluate the transmission of data at different distances within the coverage of 1.5 kilometers: $E 1=300, E 2=600$, $E 3=900, E 4=1200$ and E5=1500 meters. As shown in Fig. 6, two nodes were set up one of them was configured to function as a transmitter and the other one as a receiver.

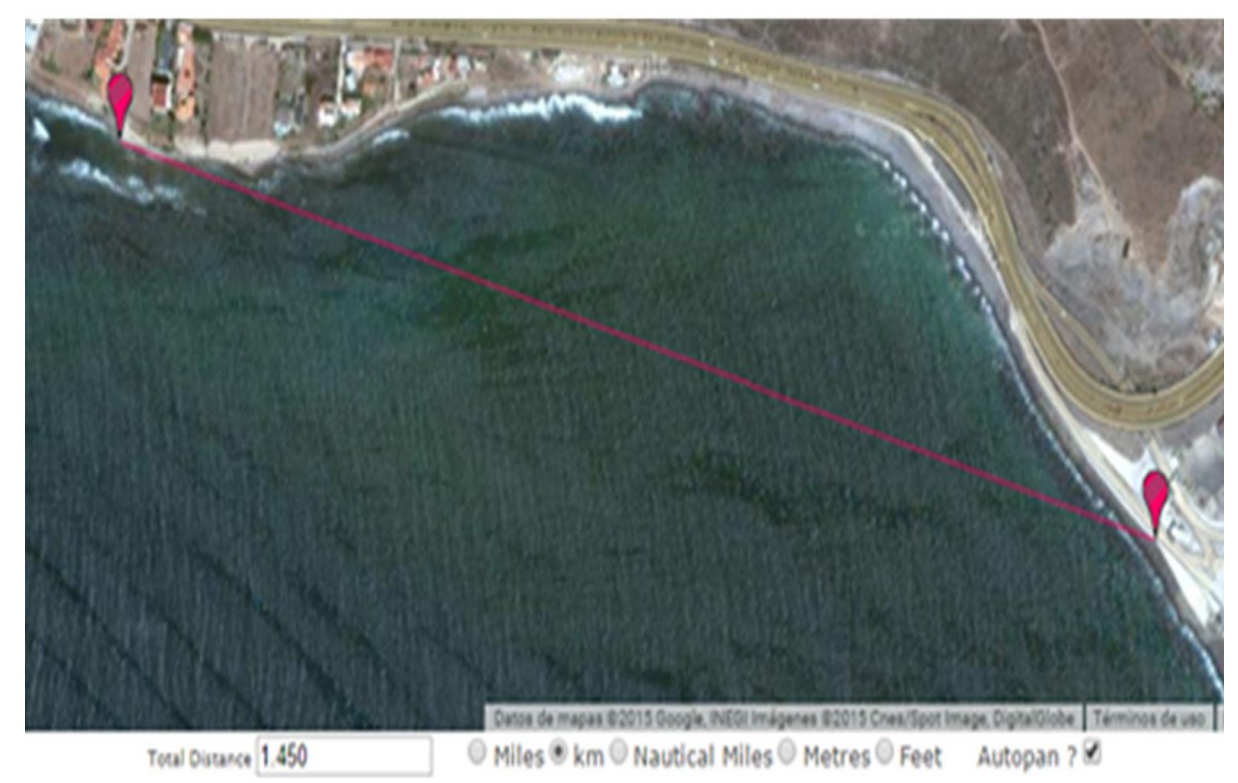

Fig. 6. Experimental scenario. 
To evaluate performance of communication, transmitter node sends 80 packets per second. Each packet consists of an 80 bytes message as recommended in [11]. Fig. 7 details the structure of the packet sent, it includes control fields, such as the type of packet, destination address node, maximum number of hops in a dissemination transmission, transmission options, checksum, among others; and information collected by the sensors or payload. The payload includes location data, speed, direction and temperature of the node.

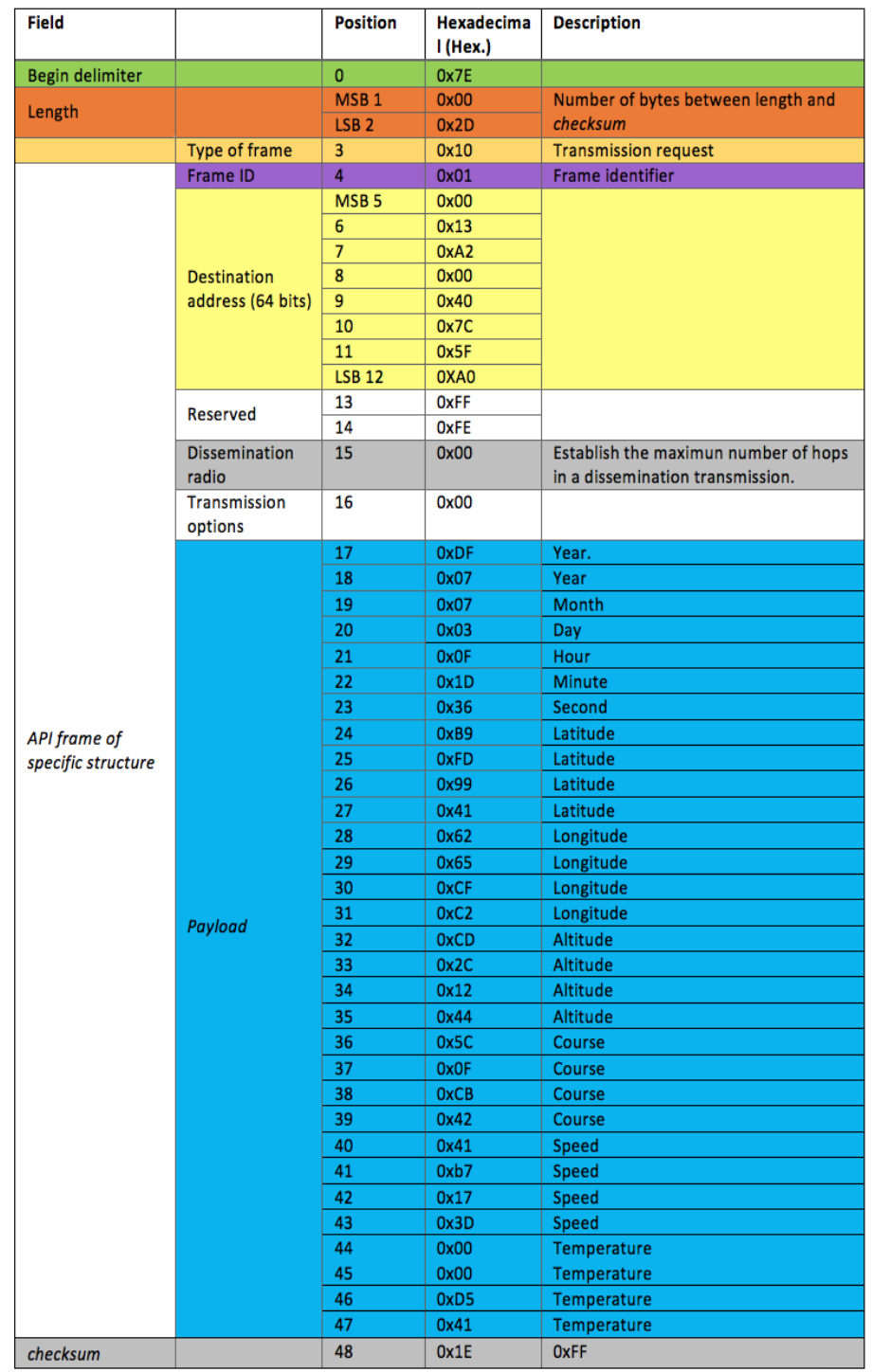

Fig. 7. Radio frequency transmission request format. 
Furthermore, to investigate transmission power necessary for warranting communication between nodes, five power settings were defined and tested: 7, 15, 18, 21 and $24 \mathrm{dBm}$.

Measurements for each setup were repeated six times. The reason for making that high number of measurements using different settings is for better estimating the behavior of node's transmissions, reducing the influence of atypical measurements. These experiments were performed randomly and concatenated to avoid bias errors as stated in [12].

Along with the record of the transmission and reception of messages, a weather station installed close to the bay (this is not part of this project) records the temperature and humidity during testing of each scenario, to analyze the effect of environmental conditions in our experiments.

Nodes utilize an algorithm, owner XBee radio, which automatically sets up power transmission level, sends packets, assigns an identifier to each packet sent, registers departure and arrival of messages and makes a test on link quality.

\subsection{Hardware used in the experiments}

Fig. 8 shows the proposed architecture. Each node is equipped with an IEEE 802.15.4 radio transmitters operating at a frequency of $900 \mathrm{MHz}$ and a development board based on Arduino Uno which is responsible for executing the different programmed experiments. Both nodes have an antenna gain of $3.5 \mathrm{dBm}$ to achieve the range of 1,500 meters. Furthermore, with the intention of reducing the number processes executed by nodes, sending node is connected to a laptop computer which is responsible for generating data packets and executing an analysis of the results obtained. The sender node is powered through a USB cable connected to the laptop computer; and the receiver node by a 9 -volt battery. The nodes were installed on a pedestal of same height for the purpose of ridding obstacles at line of sight. The height at which the antenna is placed is very important, at higher altitudes minor effects of reflection and a higher probability of packet delivery. In the work of [13] an antenna of between 1.5 and 2 meters above sea level was used. To simulate the mast where the antenna is placed on a buoy, nodes were placed on pedestals of 1.75 meters of height.

\subsection{Hardware used in the experiments}

Fig. 8 shows the proposed architecture. Each node is equipped with an IEEE 802.15.4 radio transmitters operating at a frequency of $900 \mathrm{MHz}$ and a development board based on Arduino Uno which is responsible for executing the different programmed experiments. Both nodes have an antenna gain of $3.5 \mathrm{dBm}$ to achieve the range of 1,500 meters. Furthermore, with the intention of reducing the number processes executed by nodes, sending node is connected to a laptop computer which is responsible for generating data packets and executing an analysis of the results ob- 
tained. The sender node is powered through a USB cable connected to the laptop computer; and the receiver node by a 9 -volt battery. The nodes were installed on a pedestal of same height for the purpose of ridding obstacles at line of sight. The height at which the antenna is placed is very important, at higher altitudes minor effects of reflection and a higher probability of packet delivery. In the work of [13] an antenna of between 1.5 and 2 meters above sea level was used. To simulate the mast where the antenna is placed on a buoy, nodes were placed on pedestals of 1.75 meters of height.

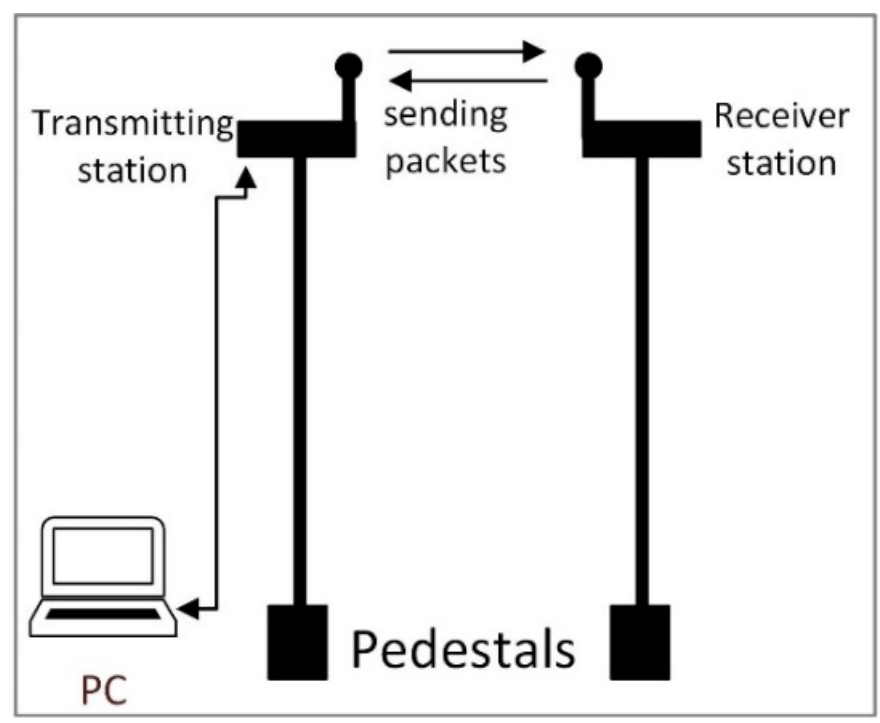

Fig. 8. Experiment setup

\subsection{Metrics evaluated}

The overall performance of a data network can be measured by evaluating different communication parameters. The most important are: received signal strength indication (RSSI), throughput (TP), round trip time (RTT) and efficiency (Ef).

RSSI estimates the link quality between two adjacent nodes. Generally, the scale is expressed in negative values and measured in decibels $(-\mathrm{dBm})$; the more negative the value, more loss of signal strength. This value is obtained directly from the XBee radio communication module through an explicit programed device. Each time a packet is generated, the device activates the program to extract the RSSI included in the packet; however, if the package is not sent because of a media access failure, the program assigns a RSSI value equal to $0 \mathrm{dBm}$. This should be taking into consideration when calculating the average of RSSI values obtained on each experiment. The IEEE standard specifies that a radio receiver may only accept signals that at least have a signal strength of $-89 \mathrm{dBm}$ or better as described in the standard [14]. 
Throughput is defined as the amount of data received during a given period of time (also known as measurement window), refer to equation (1). The TP of a system is given in bits per second and depends on attributes such as the transfer rate used and the packet size among others. Thus, having registered the beginning and end of each test and the number of bytes received at the receiving node, the throughput can be calculated [15], [16].

$$
T P=\frac{\text { Number of bits received }}{\text { measurement window }}
$$

Round Trip time is defined as the time it takes for a packet to travel from the source to the destination node and return to the source node. The main types of delays include: sending node processing delay, signal propagation delay and receiving node processing delay. Equation (2) is used to determine the RT of a package, simply calculate the difference in time between the arrival and departure times of a packet [15], [16].

$$
\text { RTT }=\text { Arrival Time }- \text { Departure time }
$$

Efficiency (Ef) of each test is defined as the number of packets received at the receiver station, represented in proportion to the total of packets sent. To calculate $E$, the number of packets received is divided by the number of packets sent during each test, as shown in equation (3).

$$
E f=\frac{\text { number of packets received }}{\text { number of packets sent }} * 100
$$

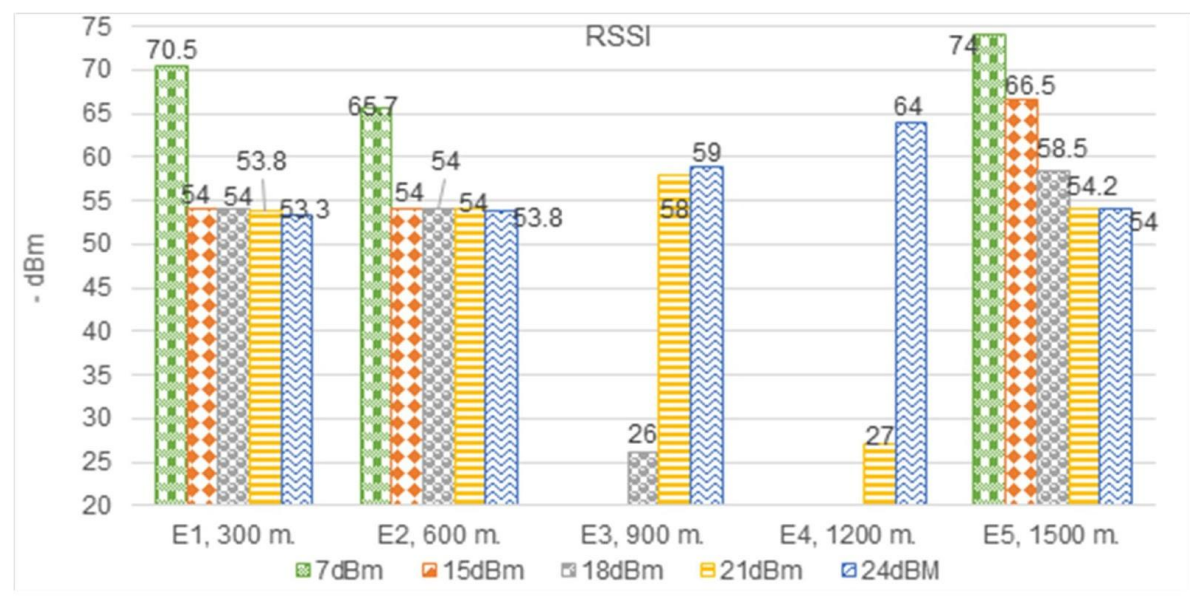

Fig. 9. RSSI obtained in each scenario. 


\section{$5 \quad$ Analysis of Results}

This section presents an analysis of the results obtained for the four transmission parameters evaluated:

\subsection{Received Signal Strength Indication (RSSI)}

In Fig. 9, RSSI values obtained using different transmission power (in $\mathrm{dBm}$ ) and distance settings (E1 to E5) is summarized. The results obtained in stages one $(300 \mathrm{~m})$ and two $(600 \mathrm{~m})$ are very similar; when using a transmission power of either 15,18 , 21 or $24 \mathrm{dBm}$ the best RSSI obtained was $-54 \mathrm{dBm}$. Better results were obtained on stages 3 and 4 using transmission distances of 900 and 1,200 meters (-26 and -27 $\mathrm{dBm}$ respectively). Notably the best results were obtained on stage 5 results of stage 5 which are better than those obtained in scenarios 3 and 4 even though a longer transmission distance was set up. On stage 5, nodes were placed 1 meter higher than in the other stages reducing the effects of reflection and attenuation of the transmitted signal because of its proximity with the surface of the sea and therefore obtaining better performance. Results demonstrated that transmitting at a distance of 1,500 meters using a transmission power of either 21 or $24 \mathrm{dBm}$ is possible.

\subsection{Throughput (TP)}

The results of TP evaluation are shown in Fig. 10. It can be seen that the best results of packet delivery to the receiver node in scenarios 1, 2 and 3 are around 4.4 Kbps when there is a good quality of the wireless link. When setting up a longer distance and reducing transmission power the quality of the signal decline and data transfer rates are lower, between 1.13 and $2 \mathrm{Kbps}$. The lowest throughput values for scenarios 1 and 2 were obtained when transmitting at $7 \mathrm{dBm}$. In scenarios 3 and 4 when transmitting at $18 \mathrm{dBm}$ throughput values were as low as $350 \mathrm{bps}$. On stage 5, throughput values of up to $4.47 \mathrm{kbps}$ were obtained, although, as previously mentioned, it should be taken into consideration that measurements at this stage were taken placing transmitters at a higher altitude.

\subsection{Round Trip Time (RTT)}

Fig. 11 shows an analysis of RTT delays. It can be concluded that when the link has good RSSI level, the best values for RTT delays are achieved; around 110 milliseconds in all stages. When the RSSI is of medium strength, RT delays range between 130 and 400 milliseconds. Whereas in low quality links (eg. stage 1, 2 and 5 with 7 $\mathrm{dBm}$ and; stage 3 and 4 with 18 and $21 \mathrm{dBm}$ ) delays increase significantly up to 2.3 seconds. This demonstrates on one hand, that the propagation delay is negligible compared with the delays generated by the nodes in the packet processing, and on the 
other hand that RT delays increase because of loss of packets and their consequently retransmission.

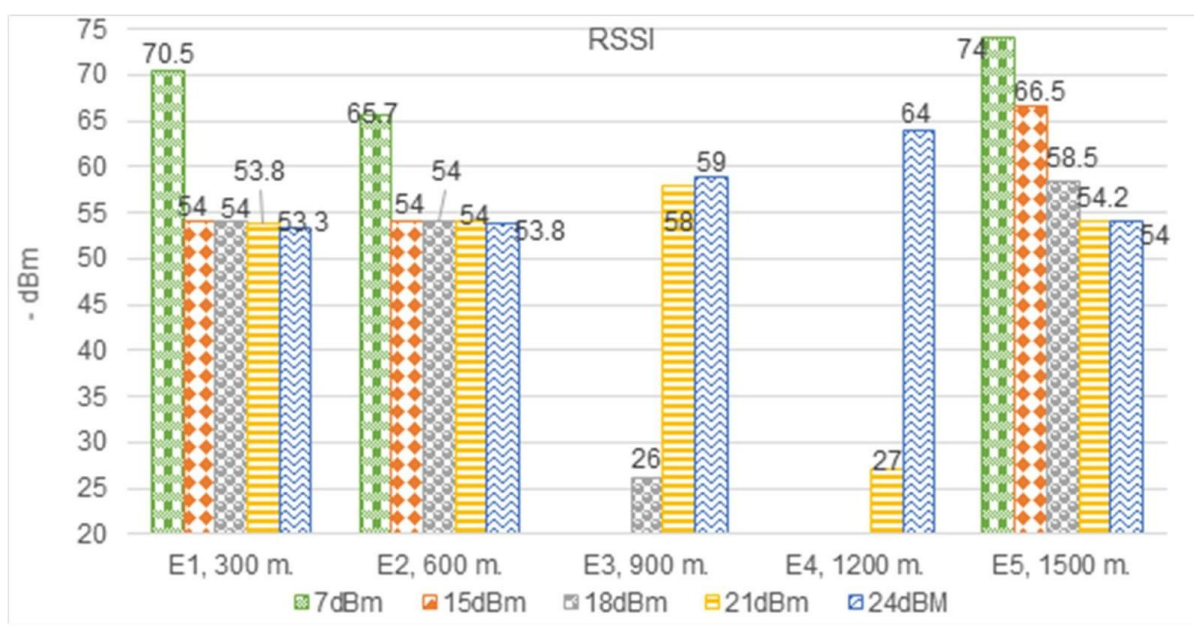

Fig. 10.RSSI obtained in each scenario.

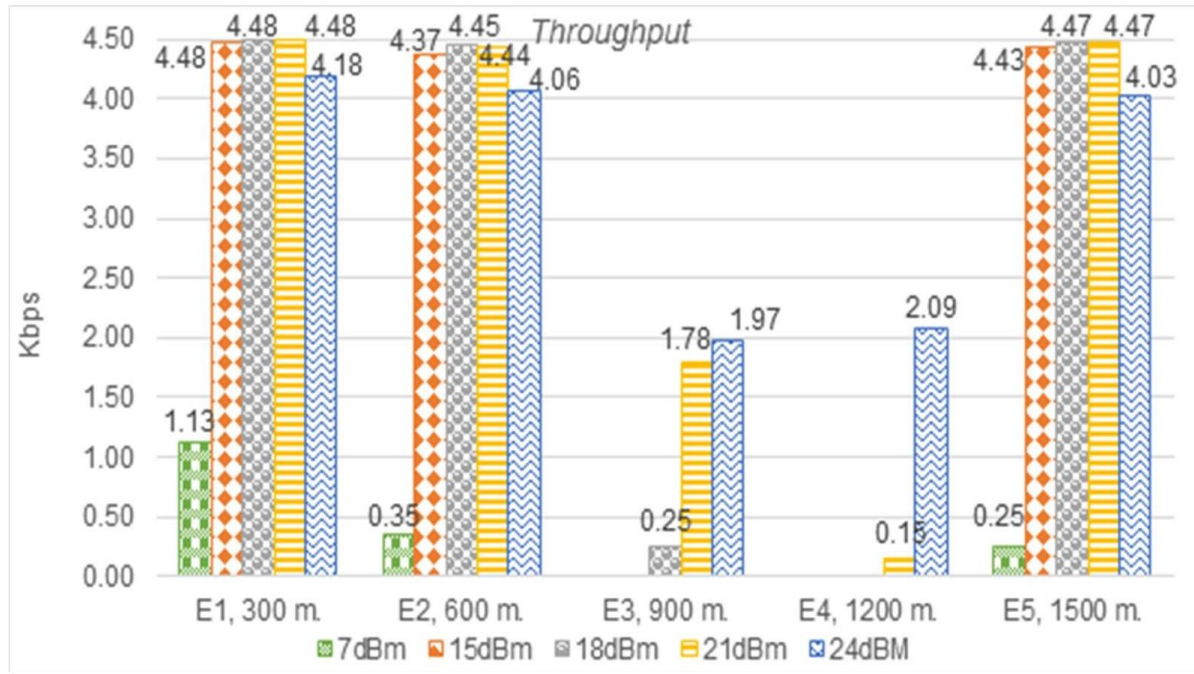

Fig. 11.Throughput achieved in experiments. 


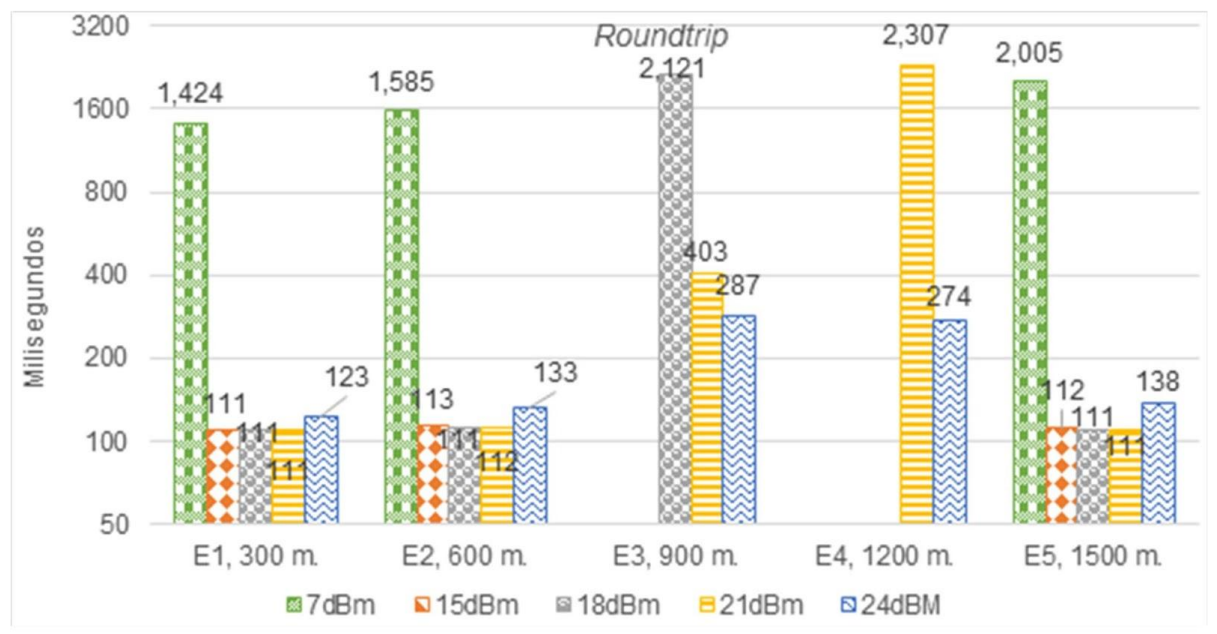

Fig. 12. Round trip delay.

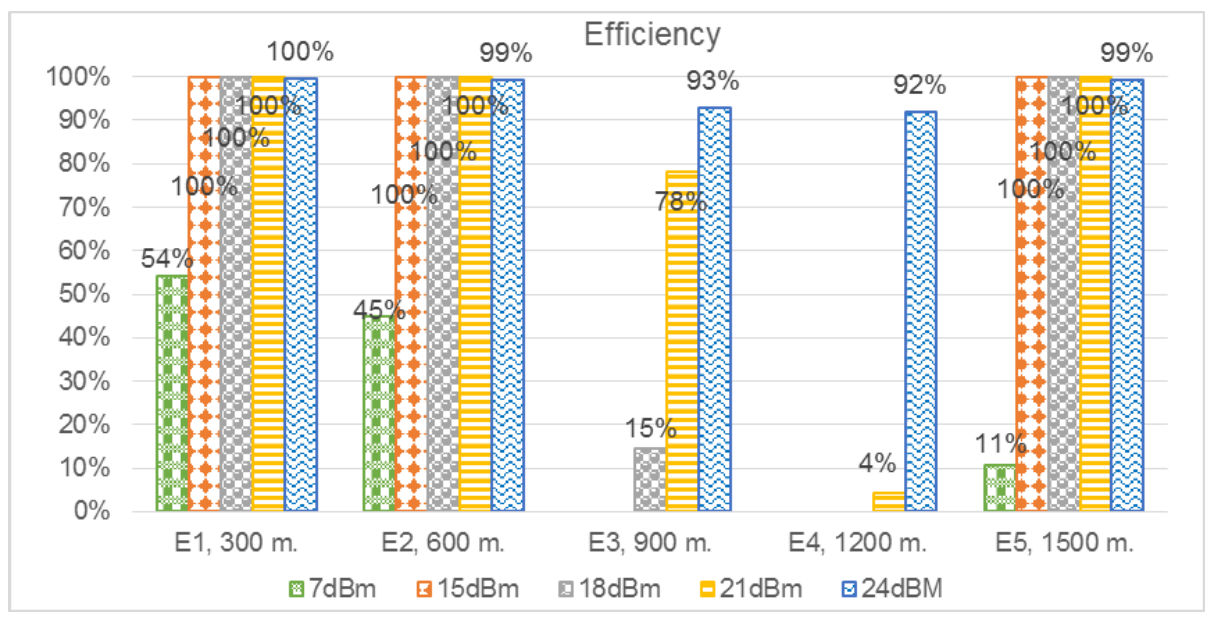

Fig. 13.Efficiency data communication.

\subsection{Efficiency (Ef)}

This metric is highly important as it determines performance of nodes in terms of data transmission. As shown in Fig. 12, the higher the transmission power the higher the efficiency obtained. In most cases a $100 \%$ of efficiency was achieved. Results also demonstrated that distance transmission between nodes has effects on the efficiency obtained. It can be observed that the longer the distance between nodes the lower the efficiency obtained. This is more evident when using lower transmission power. 
Again, on stage 5 results improve because of the placement of nodes at a higher distance from the floor.

\section{Conclusions}

The results obtained demonstrated deploying a wireless sensor network on a coastal area using the 802.15 .4 for supporting mobile communication between nodes is possible. To achieve best results the height the antenna is very important. Consequently, the design of the buoy must be analyzed carefully, since the height of the mast is determinant for achieving communication between nodes. A very high mast could compromise the stability and drag of the buoy whereas a very low mast could compromise communication. Finally, it can be concluded that the effects of the environment of coastal environments (which has high levels of temperature, relative humidity and strong winds) did not affect the performance of data transmissions between nodes. For our future work, we foresee the construction of several nodes based on the proposed architecture and deploying a complete wireless sensor network for monitoring coastal currents remotely and at real time.

\section{$7 \quad$ References}

1. J. S. Kermez and R. M. S. Mata, "DOS DÉCADAS DE HISTORIA," México, DF. 2012.

2. S. DIGAOHM, Dirección General de Oceanografía Hidrografía y Meteorología, México, 2014.

3. G. Xu, W. Shen and X. Wang, "Applications of Wireless Sensor Networks in Marine Environment Monitoring: A Survey,” in Sensors, vol. 14, no 9, pp. 16932-2014.

4. C. Albaladejo, P. Sánchez, A. Iborra, F. Soto, J. López and R. Torres, "Wireless Sensor Networks for Oceanographic Monitoring: A Systematic Review," in Sensors, vol. 10, no 7, pp. 6948, July 2010.

5. C. Zujue, G. Qiuyue and S. Zhan, "Design of WSN node for water pollution remote monitoring," in Telecommunication Systems, vol. 53, no 1, pp. 155-162, June 2013. https://doi.org/10.1007/s11235-013-9689-y

6. Y. Li, Z. Zhang, W. Huangfu, X. Chai, X. Zhu and H. Zhu, "Sea route monitoring system using wireless sensor network based on the data compression algorithm," in China Communications, vol. 11, no 13, pp. 179-186, Supplement 2014.

7. J. Guerrero, L. Garcia, J. Contreras, R. Buenrostro and M. Cosio, "HYRMA: A hybrid routing protocol for monitoring of marine environments," in IEEE Latin America Transactions, vol. 13, no 5, pp. 1562-1568, May 2015 https://doi.org/10.1109/TLA.2015.7112016

8. Y. Le, J. Ménard, C. Toquin, J. Jolivet and F. Nicolas, "Experimental measurements of propagation characteristics for maritime radio links," Intelligent Transport Systems Telecommunications,(ITST),2009 9th International Conference on, 2009, pp. 364-369.

9. J. Palmer, N. Yuen, J. Ore, C. Detweiler and E. Basha, "On air-to-water radio communication between UAVs and water sensor networks," 2015 IEEE International Conference on Robotics and Automation (ICRA), Seattle, WA, 2015, pp. 5311-5317. https://doi.org/10.1109/ICRA.2015.7139940 
10. V. Toldov, R. Igual-Pérez, R. Vyas, A. Boé, L. Clavier and N. Mitton, "Experimental evaluation of interference impact on the energy consumption in Wireless Sensor Networks," 2016 IEEE 17th International Symposium on A World of Wireless, Mobile and Multimedia Networks (WoWMoM), Coimbra, 2016, pp. 1-6.

11. R. Buenrostro, J. Nieto, J. Guerrero, M. Cosio, M. Velázquez and J. Sánchez, “Análisis de Factores que Afectan el QoS que Ofrecen las WSN Aplicado a Entornos de Salud," in Difu100cia, vol. 7, no 2, pp. 54-62, December 2013.

12. H. Gutiérrez and R. De la Vara, "Análisis y diseño de experimentos", first ed., M. Interamericana, Ed., México, 2008.

13. C. Albaladejo, F. Soto, R. Torres, P. Sánchez and J. López, "A Low-Cost Sensor Buoy System for Monitoring Shallow Marine Environments," in Sensors, vol. 12, pp. 96139634, July 2012.

14. L. M. S. Committee y I. Computer, IEEE Standard for Information technology- Telecommunications and information exchange between systems- Local and metropolitan area networks Specific requirements-Part 15.4: Wireless MAC and PHY Specifications for Low-Rate WPANs Control, New York, USA, 2006.

15. J. Birla and B. Sah, "Performance Metrics in Ad-hoc Network," in International Journal of Latest Trends in Engineering and Technology (IJLTET), vol. 1, no 1, pp. 46-49, May 2012.

16. R. Piyare and S. ro Lee, "Performance Analysis of XBee ZB Module Based Wireless Sensor Networks," in International Journal of Scientific \& Engineering Research, vol. 4, no 4, pp. 1615-1621, April 2013.

\section{Authors}

C Flores-Cortés is a Full-time Lecturer and Researcher at the University of Colima in México (UC). His research interests include distributed systems, wireless sensor networks, the internet of things, service discovery middleware, pervasive computing and mobile data applications. He received his $\mathrm{PhD}$ degree in Computing from Lancaster University in UK. Contact him at the Faculty of Telematics, UC, Av. Universidad 333, Post Code 28040, Colima, México, (cfcortes@ucol.mx).

R. Buenrostro-Mariscal is a Full-time Lecturer and Researcher at the University of Colima in México. His research interests include wireless sensor networks, internet of things, QoS provision, embedded programming, transport and MAC protocols. He received his $\mathrm{PhD}$ degree in Computing from Autonomous University of Baja California, Mexico. Contact him at the Faculty of Telematics, University of Colima, Av. Universidad 333, Post Code 28040, Colima, México, (raymundo@ucol.mx).

J. A. Guerrero-Ibáñez received his M.Sc. degree from the University of Colima (UDC), Colima, México in 1999, and the Ph.D. degree in telematics engineering from the Universitat Politècnica de Catalunya (UPC), Barcelona, in 2008. He is a full-time lecturer, researcher and a member of the Telecommunication and Networks group in the School of Telematics at the University of Colima. His research interests include networking and QoS provision, wireless sensor networks, and vehicular ad hoc networks. Contact him at the Faculty of Telematics, University of Colima, Av. Universidad 333, Post Code 28040, Colima, México, (antonio_guerrero@ucol.mx). 
F. Estrada-González is a Full-time Lecturer and Researcher and a member of the Telecommunication and Networks group in the faculty of telematics at the University of Colima in México. His research interests include computer architectures, embedded systems, embedded control, wireless sensor networks, and the internet of things. He received his M.Sc. degree in Computing from University of Colima. Contact him at the Faculty of Telematics, Av. Universidad 333, Post Code 28040, Colima, México, (fermin_estrada@ucol.mx)

J. Sandoval-Orozco received his M.Sc. degree in Computing University of Coli$\mathrm{ma}$ in 2016. His research interests include wireless sensor networks, embedded systems, automatic control and instrumentation. Contact him at (jsandoval17@ucol.mx)

This work was supported by the Program for Professional Teaching Development (ProDEP) under grant DSA/103.5/15/11080.

Submitted 03 October 2016. Published as resubmitted by the authors 16 December 2016. 